\title{
New trends in system identification
}

\author{
Kyushu University Kiyoshi WADA
}

Email: wada@ees.kyushu-u.ac.jp

In this paper we discuss three topics of system identification such as multivariable system identification (Subspace identification methods), identification for control (Robust identification), and nonlinear system identification (Nonlinear black-box modeling).

\section{Introduction}

Recent major topics in system identification include: System identification for robust control, Identification of nonlinear systems, Subspace system identification, Continuous-time identification, and Time varying system identification etc. Among them we select and review three topics, i.e., Subspace system identification, Robust identification, and Nonlinear system identification.

\section{Subspace methods}

\subsection{Problem formulation}

We assume that a linear discrete time system which is unknown can be described by the following state-space model

$$
\begin{aligned}
x_{k+1} & =A x_{k}+B u_{k} \\
y_{k} & =C x_{k}+D u_{k}+v_{k}
\end{aligned}
$$

where $x_{k}$ is an $n$-dimensional state vector, $u_{k}$ an $m$-dimensional input vector, and $y_{k}$ an $\ell$ dimensional output vector. We also assume that the system is observable and $v_{k}$ be a zeromean white stationary random process uncorrelated with the input $u_{k}$.

Define the stacked output vector $y_{i}(k)$ as

$$
y_{i}(k) \triangleq\left[\begin{array}{llll}
y_{k}^{T} & y_{k+1}^{T} & \cdots & y_{k+i-1}^{T}
\end{array}\right]^{T} \text { for } i \geq n+1 .
$$

The stacked vectors of the input and noise vectors are defined similarly. From (1), the following relations are easily derived:

$$
y_{i}(k)=\Gamma_{i} x_{k}+H_{i} u_{i}(k)+v_{i}(k)
$$

$$
x_{k}=A^{i} x_{k-i}+\Delta_{i} u_{i}(k-i)
$$

where

$$
\Gamma_{i}=\left[\begin{array}{c}
C \\
C A \\
\vdots \\
C A^{i-1}
\end{array}\right]
$$

$$
H_{i}=\left[\begin{array}{cccc}
D & & & 0 \\
C B & D & & \\
\vdots & \vdots & \ddots & \\
C A^{i-2} B & C A^{i-3} B & \cdots & D
\end{array}\right]
$$

$\Gamma_{i}$ is called an extended observability matrix. The reversed extended controllability matrix $\Delta_{i}$ is defined as

$$
\Delta_{i}=\left[A^{i-1} B, A^{i-2} B, \cdots, B\right] .
$$

From (2) and (3), we obtain

$$
\begin{aligned}
Y_{p} & =\Gamma_{i} X_{p}+H_{i} U_{p}+V_{p} \\
Y_{f} & =\Gamma_{i} X_{f}+H_{i} U_{f}+V_{f} \\
X_{f} & =A^{i} X_{p}+\Delta_{i} U_{p}
\end{aligned}
$$

where matrices $Y_{p}, Y_{f}, X_{p}$, and $X_{f}$ are defined as follows:

$$
\begin{aligned}
& Y_{p}=\left[y_{i}(0) y_{i}(1) \cdots y_{i}(N-1)\right] \quad(\ell i \times N) \\
& Y_{f}=\left[y_{i}(i) y_{i}(i+1) \cdots y_{i}(i+N-1)\right] \\
& X_{p}=\left[\begin{array}{llll}
x_{0} & x_{1} & \cdots & x_{N-1}
\end{array}\right] \quad(n \times N) \\
& X_{f}=\left[\begin{array}{llll}
x_{i} & x_{i+1} & \cdots & x_{i+N-1}
\end{array}\right] \quad(n \times N)
\end{aligned}
$$

$U_{p}, V_{p}$ and $U_{f}, V_{f}$ are defined in a similar manner as $Y_{p}$ and $Y_{f}$, respectively. 


\subsection{The Algorithm}

One of the typical algorithms of subspace identification[1] is as follows:

(step 1)

Form a matrix and perform QR-factorization as

$$
\left[\begin{array}{c}
U_{f} \\
\Phi \\
Y_{f}
\end{array}\right]=\left[\begin{array}{lll}
R_{11} & & \\
R_{21} & R_{22} & \\
R_{31} & R_{32} & R_{33}
\end{array}\right]\left[\begin{array}{c}
Q_{1}^{T} \\
Q_{2}^{T} \\
Q_{3}^{T}
\end{array}\right]
$$

where $R_{11}, R_{22}$, and $R_{33}$ are some lower triangular matrices, and $\left[\begin{array}{lll}Q_{1} & Q_{2} & Q_{3}\end{array}\right]$ is a matrix whose column vectors are orthonormal.

(step 2)

Compute Singular Value Decomposition (SVD) of $R_{32}$ as follows:

$$
R_{32}=\left[\begin{array}{ll}
E_{n} & E_{n}^{\perp}
\end{array}\right]\left[\begin{array}{ll}
S_{n} & \\
& S_{2}
\end{array}\right]\left[\begin{array}{c}
F_{n}^{T} \\
\left(F_{n}^{\perp}\right)^{T}
\end{array}\right]
$$

$\underline{\text { (step 3) }}$ Let $\widehat{A}=\left(E_{n}^{(1)}\right)^{\dagger}\left(E_{n}^{(2)}\right)$ and $\widehat{C}$ be the first $\ell$ rows of the $E_{n}$. Here $E_{n}^{(1)}$ and $E_{n}^{(2)}$ consist of first and last $\ell \times(i-1)$ rows of $E_{n}$, respectively.

(step 4) Compute $\widehat{B}$ and $\widehat{D}$ solving a certain set of linear equations.

In the sequel we explore the implication of the algorithm.

\subsection{Multivariate Linear Regres- sion}

Denoting by $\Gamma_{i}^{\dagger}$ the Moore-Penrose psuedoinverse of $\Gamma_{i}$, it follows from (4) that

$$
X_{p}=\Gamma_{i}^{\dagger}\left[Y_{p}-H_{i} U_{p}-V_{p}\right] .
$$

Thus substituting the above into (6), we get

$$
X_{f}=K \Phi-A^{i} \Gamma_{i}^{\dagger} V_{p}
$$

where

$$
K=\left[\Delta_{i}-A^{i} \Gamma_{i}^{\dagger} H_{i} \mid A^{i} \Gamma_{i}^{\dagger}\right], \Phi=\left[\begin{array}{c}
U_{p} \\
Y_{p}
\end{array}\right] .
$$

Substituting the $X_{f}$ into (5), we see that $Y_{f}$ can be expressed as

$$
Y_{f}=L_{i} \Phi+H_{i} U_{f}+V_{f}-A^{2 i} \Gamma_{i}^{\dagger} V_{p}
$$

where $L_{i}=\Gamma_{i} K$.

Consider the problem minimizing the following criterion for $L_{i}$ and $H_{i}$, regarding the (10) as a multivariate linear regression :

$$
\left\|Y_{f}-\left(L_{i} \Phi+H_{i} U_{f}\right)\right\|_{F}^{2}
$$

where $\|\cdot\|_{F}$ denotes the Frobenius norm. $\widehat{H}_{i}$ and $\widehat{L}_{i}$ minimizing the above criterion are

$$
\begin{aligned}
& \widehat{H}_{i}=\left(Y_{f}-\widehat{L} \Phi\right) U_{f}^{T}\left(U_{f} U_{f}^{T}\right)^{-1} \\
& \widehat{L}_{i}=Y_{f} \Pi_{U_{f}}^{\perp} \Phi^{T}\left(\Phi \Pi_{U_{f}}^{\perp} \Phi^{T}\right)^{-1}
\end{aligned}
$$

respectively, where $\Pi_{U_{f}}^{\perp}$ is defined as

$$
\Pi_{U_{f}}^{\perp}=I-U_{f}^{T}\left(U_{f} U_{f}^{T}\right)^{-1} U_{f} .
$$

Using the $R Q$ decomposition, the criterion (11) can be rewritten as

$$
\begin{gathered}
\left\|Y_{f}-\left(L_{i} \Phi+H_{i} U_{f}\right)\right\|_{F}^{2} \\
=\left\|\left[R_{31}, R_{32}\right]-\left[H_{i} L_{i}\right]\left[\begin{array}{ll}
R_{11} & \\
R_{21} & R_{22}
\end{array}\right]\right\|_{F}^{2} \\
+\left\|R_{33}\right\|_{F}^{2} .
\end{gathered}
$$

Thus, the estimates $\widehat{L}_{i}$ and $\widehat{H}_{i}$ are the solutions of the following equation

$$
\left[R_{31}, R_{32}\right]=\left[\widehat{H}_{i} \widehat{L}_{i}\right]\left[\begin{array}{ll}
R_{11} & \\
R_{21} & R_{22}
\end{array}\right] .
$$

It follows from this equation that the estimate $\widehat{L}_{i}$ satisfies a relation

$$
\widehat{L}_{i} R_{22}=R_{32} \text {. }
$$

\subsection{Estimation of extended ob- servability matrix $\Gamma_{i}$}

To understand a fundamental notion of what the subspace methods are, it is relevant to recognize the extended observability matrix $\Gamma_{i}$ plays a central role in the method.

It has been proposed[1] that SVD of the quantity $R_{32}$ shown in (8) is utilized to obtain the extended observability matrix $\Gamma_{i}$ as in

$$
\Gamma_{i}=E_{n}
$$


This section discusses why SVD of $R_{32}$ leads to computation of $\Gamma_{i}$.

$U_{f}=R_{11} Q_{1}^{T}$ follows from $Q R$ factorization, and then

$$
\Pi_{U_{f}}=U_{f}^{T}\left(U_{f} U_{f}^{T}\right)^{-1} U_{f}=Q_{1} Q_{1}^{T}
$$

and we see also

$$
\Pi_{U_{f}}^{\perp}=I-Q_{1} Q_{1}^{T}
$$

Furthermore since $\Phi=R_{21} Q_{1}^{T}+R_{22} Q_{2}^{T}$, we have

$$
\Phi \Pi_{U_{f}}^{\perp}=R_{22} Q_{2}^{T} .
$$

Postmultiplying both side of (13) by $Q_{2}^{T}$, we obtain the relation

$$
\widehat{L}_{i} \Phi \Pi_{U_{f}}^{\perp}=R_{32} Q_{2}^{T} .
$$

On the other hand, from (5) and noting $U_{f} \Pi_{U_{f}}^{\perp}=0$, we obtain

$$
\begin{aligned}
\widehat{L}_{i} \Phi \Pi_{u_{f}}^{\perp} & =\Gamma_{i} X_{f} \Pi_{U_{f}}^{\perp} \Phi^{T}\left(\Phi \Pi_{U_{f}}^{\perp} \Phi^{T}\right)^{-1} \Phi \Pi_{U_{f}}^{\perp} \\
& +V_{f} \Pi_{U_{f}}^{\perp} \Phi^{T}\left(\Phi \Pi_{U_{f}}^{\perp} \Phi^{T}\right)^{-1} \Phi \Pi_{U_{f}}^{\perp} .
\end{aligned}
$$

By assumption

$$
\begin{aligned}
& \lim _{N \rightarrow \infty} \frac{1}{N} V_{f} U_{f}^{T}=0 \\
& \lim _{N \rightarrow \infty} \frac{1}{N} V_{f} \Phi^{T}=0
\end{aligned}
$$

so we have

$$
\lim _{N \rightarrow \infty} \frac{1}{N} V_{f} \Pi_{U_{f}}^{\perp} \Phi^{T}=0
$$

and for sufficiently large $N$ we can verify that

$$
\widehat{L}_{i} \Phi \Pi_{U_{f}}^{\perp} \approx \Gamma_{i} X_{f} \Pi_{U_{f}}^{\perp} \Phi^{T}\left(\Phi \Pi_{U_{f}}^{\perp} \Phi^{T}\right)^{-1} \Phi \Pi_{U_{f}}^{\perp} .
$$

Note that both the left singular vectors of $R_{32}$ and $R_{32} Q_{2}^{T}$ are identical since $Q_{2}^{T} Q_{2}=I$. Therefore we can obtain $\Gamma_{i}$ via SVD of $R_{32}$ for sufficiently large $N$.

Above discussions lead to a conjecture that SVD of not only $\widehat{L}_{i} \Phi \Pi_{U_{f}}^{\perp}$, but also of $\widehat{L}_{i} \Phi$, again of $Y_{f} \Pi_{U_{f}}^{\perp} \Phi^{T}$ can lead to $\Gamma_{i}$ according to the property of (16). In fact, we can see [2] that
N4SID method is a version of subspace method based on SVD of

$$
\widehat{L}_{i} \Phi=Y_{f} \Pi_{U_{f}}^{\perp} \Phi^{T}\left(\Phi \Pi_{U_{f}}^{\perp} \Phi^{T}\right)^{-1} \Phi
$$

Furthermore in PI-MOESP methods [3], we obtain $\Gamma_{i}$ via SVD of

$$
Y_{f} \Pi_{U_{f}}^{\perp} U_{p}^{T}
$$

while, replacing $U_{p}$ with $\Phi$ in the above equation, we can see [6] it is no other than a subspace method based on SVD of

$$
Y_{f} \Pi_{U_{f}}^{\perp} \Phi^{T}=R_{32} R_{22}^{T} .
$$

To concluding this section, we point out that different versions of subspace methods correspond to various estimates of $\Gamma_{i}$ 's; but all the estimates are essentially represented as

$$
\Gamma_{i}=E_{n} T_{s}
$$

where $T_{s}$ is an arbitrary real nonsingular matrix. We should stress that one of the most principal point of subspace methods is extracting the extended observability matrix from input-output data perturbed by noise.

\section{Robust identification [4]}

Assume that a linear discrete time SISO system can be described by the following transfer function $G^{0}(z)$

$$
G^{0}(z)=G(z)+\Delta(z)
$$

where $\Delta(z)$ denotes an additive uncertainty, and $G(z)$ a nominal model represented by

$$
G(z)=\frac{b(z)}{a(z)}=\frac{b_{0}+b_{1} z^{-1}+\cdots+b_{n} z^{-n}}{1+a_{1} z^{-1}+\cdots+a_{n} z^{-n}}
$$

where $z$ is a forward shift operator.

Consider a minimization problem of $\mathcal{H}_{\infty}$ norm of the additive uncertainty $\Delta(z)$ :

$$
J=\|\Delta(z)\|_{\infty} .
$$

subject to the condition that the model can explain some given input and output 


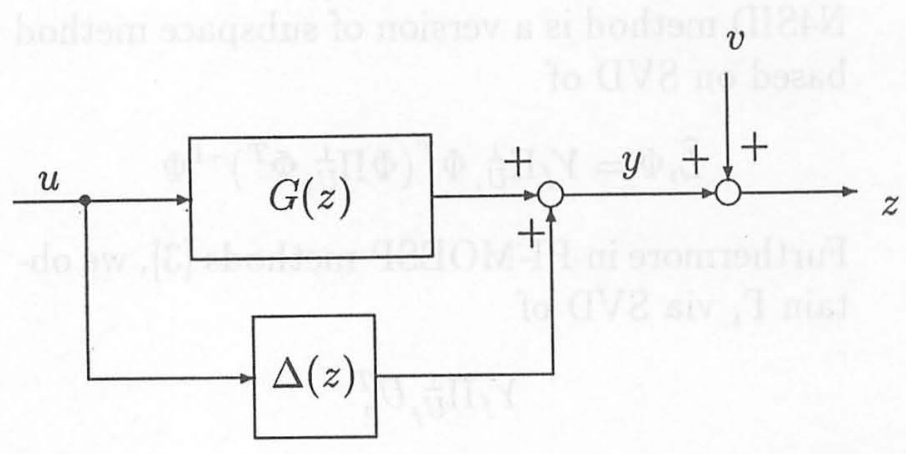

Fig. 1 The System

data. We can approach the problem with the Carathéodory-Fejér theorem:

$\ll$ Theorem $1 \gg$

$$
\begin{aligned}
\min _{p(z) \in \mathcal{H}_{\infty}} & \left\|f_{0}+\cdots+f_{N-1} z^{-(N-1)}+z^{-N} p(z)\right\|_{\infty} \\
= & \left\|f_{0}+f_{1} z^{-1}+\cdots+f_{N-1} z^{-(N-1)}\right\|_{H} \\
= & \bar{\sigma}\left(F_{N}\right)
\end{aligned}
$$

where $\bar{\sigma}(\cdot)$ represents the maximum singular value of $(\cdot)$, and $N \times N$ Toeplitz matrix $F_{N}$ is defined as

$$
\left[\begin{array}{cccc}
f_{0} & & & O \\
f_{1} & f_{0} & & \\
\vdots & \ddots & \ddots & \\
f_{N-1} & \cdots & f_{1} & f_{0}
\end{array}\right]
$$

for a. function " $F(z)$ " $\left(f_{0}+f_{1} z^{-1}+f_{2} z^{-2}+\cdots\right)$ or signal " $f$ " $\left(f_{0}, f_{1}, f_{2}, \cdots\right)$. For the signals $y, u$, and transfer function $G^{0}(z)$, we denote the corresponding Toeplitz matrices by $Y_{N}, U_{N}$ and $G_{N}^{0}$, respectively. Then we have $Y_{N}=G_{N}^{0} U_{N}$, and thus

$$
G_{N}^{0}=Y_{N} U_{N}^{-1}
$$

provided $u_{0} \neq 0$. Since $G^{0}(z)=G(z)+\Delta(z)$ from (18), denoting the Toeplitz matrices of $G(z)$ and $\Delta(z)$ by $G_{N}$ and $\Delta_{N}$, respectively, we see

$$
\Delta_{N}=G_{N}^{0}-G_{N}
$$

and $G_{N}=\mathcal{B}_{N} \mathcal{A}_{N}^{-1}$ from (19), where $\mathcal{B}_{N}$ and $\mathcal{A}_{N}$ are the Toeplitz matrices for $b(z)$ and $a(z)$, respectively. Using above equation, we have

$$
\Delta_{N}=Y_{N} U_{N}^{-1}-\mathcal{B}_{N} \mathcal{A}_{N}^{-1} .
$$

Thus, the polynomial coefficients of $\Delta_{N}$, $\delta_{0}, \delta_{1}, \cdots, \delta_{N-1}$, are obtained up to $N-1$, and we can see from Theorem 1 that

$$
\begin{aligned}
& \min _{p(z) \in \mathcal{H}_{\infty}}\left\|\delta_{0}+\cdots+\delta_{N-1} z^{-(N-1)}+z^{-N} p(z)\right\|_{\infty} \\
& =\bar{\sigma}\left(\Delta_{N}\right)=\bar{\sigma}\left(Y_{N} U_{N}^{-1}-\mathcal{B}_{N} \mathcal{A}_{N}^{-1}\right)
\end{aligned}
$$

$\ll$ Theorem 2 》

Assume that the system has no noise and be with zero initial condition. Given the inputoutput time series data $\left(u_{i}, y_{i}, u_{0} \neq 0\right)$, an error function $\Delta(z)$ exists such that $\|\Delta(z)\|_{\infty} \leq \varepsilon$ if and only if the following condition holds:

$$
\bar{\sigma}\left(\Delta_{N}\right)=\bar{\sigma}\left(Y_{N} U_{N}^{-1}-\mathcal{B}_{N} \mathcal{A}_{N}^{-1}\right) \leq \varepsilon
$$

Note that if the Toeplitz matrix $\mathcal{A}_{N}$ corresponding to the denominator of the model, can be obtained via some other schemes, then $\Delta_{N}$ is a linear function of $\mathcal{B}_{N}$ which is of the numerator. A method to obtain some kind of linear relation of the denominator has been proposed via formulating a series of estimates $\bar{\sigma}\left(\Delta_{N}^{(i)}\right)$ for $\bar{\sigma}\left(\Delta_{N}\right)$ where

$$
\Delta_{N}^{(i)}=\left(\mathcal{A}^{(i-1)} U_{N}\right)^{-1}\left(\mathcal{A}^{(i)} Y_{N}-\mathcal{B}^{(i)} U_{N}\right) .
$$

$\Delta_{N}^{(i)}$ is linear with respect to $\mathcal{B}^{(i)}$ as well as $\mathcal{A}^{(i)}$ and we may solve the problem numerically by iteration.

\section{Nonlinear black-box model [5]}

In this section, for simplicity, we consider the SISO case. Collect a finite number of past inputs $u_{k}$ and outputs $y_{k}$ into the vector $\varphi_{k}$

$$
\varphi_{k}=\left[y_{k-1}, \cdots, y_{k-n}, u_{k-1}, \cdots, u_{k-n}\right]^{T}
$$

The problem then is to understand the relationship between the next output $y_{k}$ and $\varphi_{k}$ :

$$
y_{k} \leftrightarrow \varphi_{k}
$$

\section{Non-linear regression set-up}

For a particular value $\varphi_{k}$ the corresponding $y_{k}$ is assumed to be

$$
y_{k}=g_{0}\left(\varphi_{k}\right)+e_{k}
$$


where $\left\{e_{k}\right\}$ is supposed to be a sequence of independent random variables, with zero mean and variance $\sigma_{e}^{2}$. To find the function $g_{0}$ in (23) we have the following information available:

1. A parametrized family of functions

$$
\mathcal{G}_{m}=\left\{g\left(\varphi_{k}, \theta\right) \mid \mathcal{D}_{\mathcal{M}}\right\}
$$

2. A collection of observed $y_{k}, \varphi$-pairs:

$$
Z^{N}=\left\{y_{k}, \varphi_{k} \mid k=1, \cdots, N\right\}
$$

The typical way to estimate $g_{0}$ is then to form the scalar valued function

$$
J_{N}(\theta)=\frac{1}{N} \sum_{k=1}^{N}\left(y_{k}-g\left(\varphi_{k}, \theta\right)\right)^{2}
$$

and determine the parameter estimate $\widehat{\theta}_{N}$ as its minimizing argument:

$$
\widehat{\theta}_{N}=\arg \min J_{N}(\theta)
$$

The estimate of $g_{0}$ will then be

$$
\widehat{g}_{N}(\varphi)=g\left(\varphi, \hat{\theta}_{N}\right) .
$$

Usually we use function expansions of the type

$$
g(\varphi, \theta)=\sum_{k=1}^{q} \theta_{i} g_{i}(\varphi)
$$

where $\left\{g_{i}(\varphi)\right\}$ is a basis for the function $g_{0}(\varphi)$. Out of the many possible choice of basis functions, a large family of special ones have received most of the current interest. They are all based on just one fundamental function $\mu(\varphi)$, which is scaled in various ways, and centered at different points,i.e.

$$
g_{i}(\varphi)=\mu\left(\beta_{i}^{T} \varphi+\gamma_{i}\right)=\mu\left(\varphi, \eta_{i}\right) .
$$

\section{References}

[1] M. Viberg (1995) : Subspace-based Methods for the Identification of Linear Timeinvariant Systems, Automatica , 31-12, pp.1835-1851.

[2] P. Van Overschee and B. De Moor (1996) : Subspace Identification for Linear Systems - Theory Implementation Applications. Kluwer Academic Publisher.
[3] M. Verhaegen (1993) : Subspace Model Identification, Part 3, Analysis of the Ordinary Output-error State Space Model Identification Algorithm, International Journal of Control, 58-3, pp.555-586

[4] T. Zhou and H. Kimura (1994) : Simultaneous Identification of Nominal Model, Parametric Uncertainly for Robust Control. Automatica , 30-3,pp.391-402.

[5] L. Ljung, J. Sjöberg, and H. Hjalmarsson (1996) : On Neural Network Model Structures in System Identification, In S.Bittanti and G.Picci (Eds), Identification, Adaptation, Learning: The Science of Learning Models from Data, SpringerVerlag, Berlin Heiderberg.

[6] T.Ishii, J. Imai and K. Wada (1996) : A Study on 4SID method based on IV method (in Japanese), The 19th SICE Symposium on Dynamical System Theory, pp. $185-188$ 


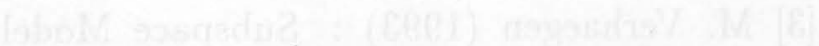

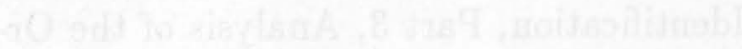

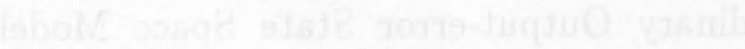

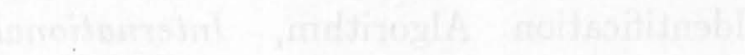

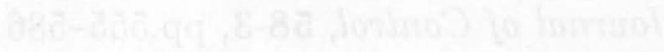

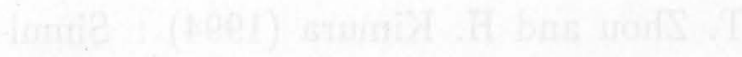

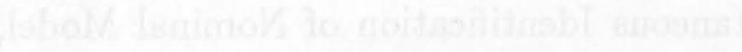

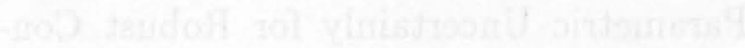

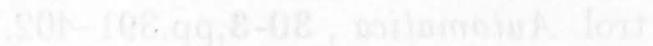

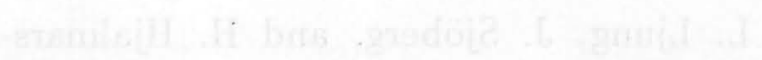

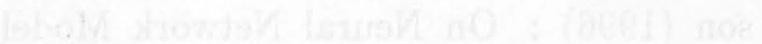

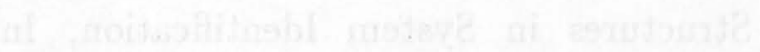

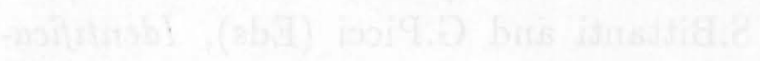

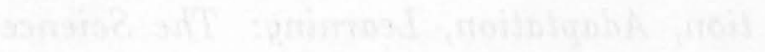

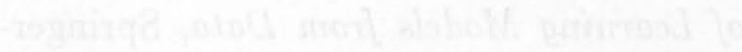

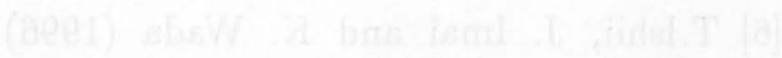

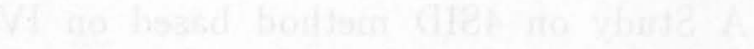

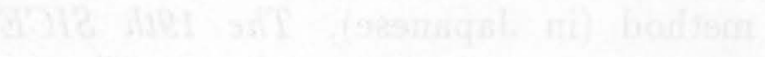

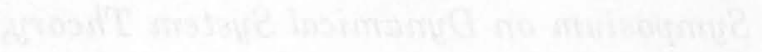

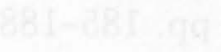

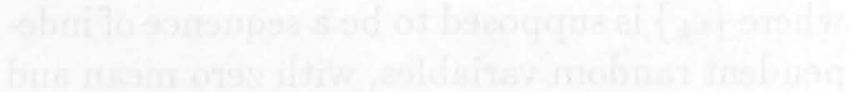
axt

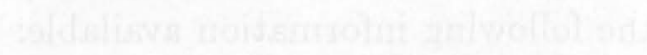

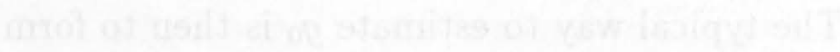

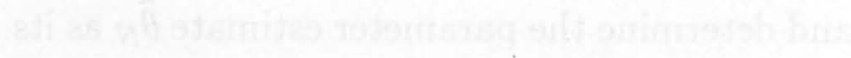

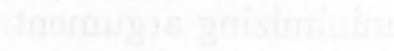

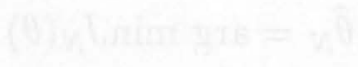

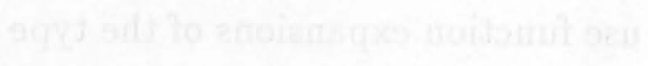

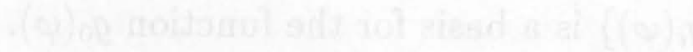

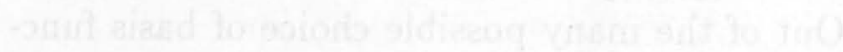

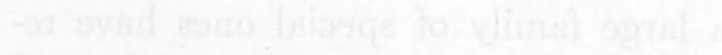

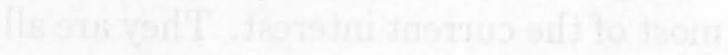

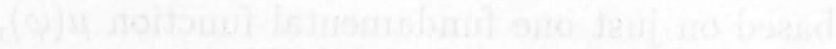

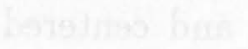

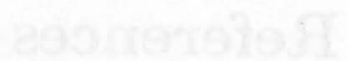

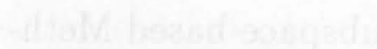

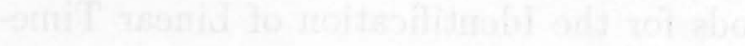

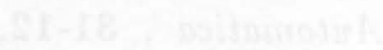

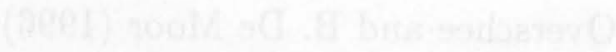

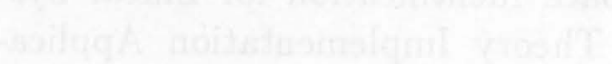

